\title{
Hacer lo que resulta natural $\left(^{*}\right)$
}

\section{R. S. Herrnstein}

Universidad de Harvard

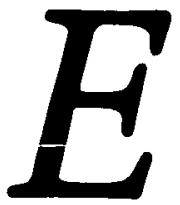

En la respuesta del profesor Skinner (diciembre de 1977) a mi artículo (Herrnstein, agosto de 1977) se emplean varios términos sin definir para referirse a un tipo de conducta. Habla de la "conducta adjuntiva", la "conducta innata", la "condúcta filogenética», el "repertorio filogenético" (de los animales y los seres humanos) y la "conducta desencadenada». Por el contexto parece que estas categorías son sinónimas o por lo menos que se superponen. Todas ellas se refieren a la "conducta debida a la selección natural", distinguiéndola de la conducta debida al condicionamiento. Ejemplos de estas conductas son las de las anguilas que emigran de "un río europeo al mar de los Sargazos" o la "respuesta exploratoria de la paloma" en el laboratorio. Aunque Skinner no define esta clase de conducta, el contexto en que se refiere a ella nos da indicios sobre el sentido en que la utiliza. Para Skinner no es primordialmente un tipo de conducta operante, reforzada, porque eso es precisamente lo que yo pretendia que era y lo que sí está claro por lo menos es que él no está de acuerdo conmigo. Pero tampoco es una conducta refleja (respondente), puesto que él divide las conductas innatas en dos clases cuando habla de las "conductas reflejas y desencadenadas». No es ni una conducta operante ni respondente, con lo que se sitúa fuera del alcance del análisis skinneriano (aunque evidentemente no esté fuera del alcance de la atención personal de Skinner).

La presencia de un tercer tipo de conductas constituye una mala nueva para la teoría skinneriana. En realidad, la convicción de que existe ha suscitado, en gran medida, ese antiskinnerianismo que señalaba en mi artículo. Como yo creía que está abogando en contra de esa tercera categoría, me resulta extraño verme en este intercambio con Skinner. Quizá no resulta evidente por qué no debería proponerse. de forma causal -y especialmente por los skinnerianos-- la existencia de una clase de conductas filogenéticas sin carácter reflejo.

¿Por qué sería esta una mala noticia para los skinnerianos? Hay varias razones que se resumen brevemente a continuación: 
1. La Teoria Skinneriana apela a dos tipos de conductas - la operante y la respondente- y a dos conjuntos de leyes que describen su forma de darse, sobre todo la ley del efecto y el condicionamiento clásico, respectivamente. $\mathrm{Si}$ existe una tercera clase de conductas, entonces la teoría carece de un conjunto de leyes que las describan.

2. La Teoría Skinneriana definió operacionalmente sus dos categorías de conducta. La conducta respondente es «la conducta que está correlacionada con estímulos específicos que la elicitan" (Skinner, 1938, p. 20). Y una operante es «una conducta tal que no está sometida a este tipo de control" (p. 20). Además "una operante es una parte identificable de la conducta. De ella no puede decirse que no pueda encontrarse un estímulo que la elicite (puede haber una respondente cuya respuesta tenga la misma topografía), sino que no puede detectarse un estímulo correlacionado en las ocasiones en que se observa» (p. 21). Parece que o bien la conducta es elicitada por estímulos en todas las ocasiones - y entonces es respondente- 0 no $-\mathrm{y}$ entonces es operante-, pero el profesor Skinner no nos dice dónde situar la conducta filogenética. La vincula a la conducta operante, en tanto en cuanto es "modelada" por la selección natural (Skinner, 1975), pero no dice lo suficiente como para definirla. Puesto que se pretendía que las dos categorias originales fueran exhaustivas, parece que Skinner no ha conseguido reconciliar esa nueva categoria de conductas con su propia teoria.

3. La Teoría Skinneriana no nos da ninguna información empírica sobre cómo interactúan estas tres clases de conductas (suponiendo, de nuevo; que haya un tercer tipo además de la operante y la respondente). Cuando se define el picoteo investigatorio de la paloma, Skinner (1977) dice que "la filo- genia y la ontogenia son amistosas rivales y ninguna de ellas vence siempre a la otra" (p. 69), lo que no nos ayuda' mucho.

4. Como cuestión práctica, la conducta filogenética puede constituir un problema grande o pequeño para los skinnerianos, dependiendo de que comprenda una fracción grande o pequeña de la conducta de un organismo. Si constituyera, por ejemplo, un $99 \%$ de la conducta, entonces los skinnerianos dispondrian de leyes para dar cuenta del $1 \%$, como mucho, de la conducta del organismo. El otro $99 \%$ respondería a leyes que aún están sin formular. Quizá parezca que esto constituye un pesimismo poco realista, pero quizá no lo sea si tenemos en cuenta que la teoría skinneriana no da cuenta ni de la conducta filogenética como tal, ni de las interacciones entre la conducta filogenética y otras clases de conductas. El problema no reside sólo en que haya alguna fracción de la conducta que se sitúa fuera del alcance de la teoría skinneriana, sino que no parece haber forma de medir su amplitud, ni se nos proporciona ninguna prueba para descubrirla. Al postular la existencia de una tercera categoría de conductas, sin dar cuenta de ellas, se plantea la cues. tión de la naturaleza-cultura en términos esencialmente pre-skinnerianos. Creo que podemos hacerlo mejor.

Mi sugerencia de evitar la carga teórica que supone proponer una tercera categoría de conductas es clara: no lo hagamos. Por lo menos, no todavia. Por el momento, las pruebas no nos exigen que demos un paso tan temerario. En mi artículo trataba de explicar por qué no, pero parece que hay ciertos argumentos que necesitan más apoyo.

Skinner se resiste a aceptar mi sugerencia de que hay ciertos estímulos que se originan en la conducta y que pueden ser autorreforzantes. Por el con- 
trario, él argumenta que la noción de auto-reforzamiento es superflua desde el punto de vista teórico: "la caza al acecho es, simplemente, el tipo de cosa que el gato hace como tal gato, y está sujeta a modificaciones considerables por virtud de reforzadores identificables a lo largo de la vida del individuo" (p. 72). La caza al acecho es diferente de la conducta reforzada, dice Skinner, situándola en la misma categoría de "las arcadas o la digestión de la comida".

Me hubiera gustado que Skinner nos hubiera dicho cómo lo sabe, porque me sorprende. Yo hubiera supuesto que un gato presionaría una palanca para obtener la oportunidad de cazar un ratón, incluso un ratón de juguete. Yo he tenido gatos a los que parecía reforzarles más dar zarpazos a un cordel que se arrastraba por el suelo que algunas de las comidas de gatos con las que les alimentaba. Con seguridad, hay algo filogenético en la caza, ¿pero, por qué no acercarnos primero al problema con el mismo sistema de análisis conductual que debemos, en gran parte, a Skinner? Skinner parece demasiado dispuesto a abandonar la formulación del problema en estos términos, y eso es lo que hace al proponer una tercera categoría de conductas.

La razón por la que abandona el problema es que algunas conductas - como la migración de la anguila y quizá la caza del gato- surgen completamente desarrolladas cuando se dan por primera vez (y a veces sólo se dan esa vez). Como Skinner concibe el reforzamiento como una ley del aprendizaje, la migración de las anguilas no puede ser reforzada. En el sistema de Skinner, la ley del efecto sirve a una doble función: produce el aprendizaje y mantiene la conducta. Por el contrario, yo creo, ahora, que lo mejor es limitar la ley del efecto a un principio de mantenimiento y no de aprendizaje y mantenimiento (Brown y Herrnstein, 1975). El que ha formulado una argumentación más concreta contra la ley del efecto como principio de aprendizaje es MacKintosh (MacKintosh, 1974), aunque también otro de los primeros discípulos de Skinner, por lo menos (Estes, 1969), está de acuerdo. Estaría fuera de lugar revisar aquí los argumentos, pero sí es preciso señalar que una de las ventajas de limitar la ley del efecto al mantenimiento de la conducta es que permite incluir, en ella, la migración de la anguila.

El profesor Skinner (1977) alude a los problemas epistemológicos que se plantean al hacerlo. Dice que "no puede haber un movimiento de ida al nuevo territorio y otro de vuelta que se deban al autorrefuerzo, porque la anguila sólo hace el viaje una vez y sólo pueden ser efectivos los refuerzos pasados. Mantener que un organismo se comporta de determinada forma en virtud de alguna consecuencia reforzante futura es una postura innecesariamente teleológica» (p. 12). Pero ¿cuál es el problema en este caso? Supongamos que la anguila tuviera que aprender la ruta migratoria, como las vacas aprenden a ir al lugar del pasto. Como cientificos naturales, tenemos que suponer que los resultados del aprendizaje se representan físicamente en la anguila, aunque no necesitemos saber cómo se representan, ni siquiera dónde. Pero si la anguila heredase la ruta migratoria, como de hecho ocurre, también tendríamos que suponer que los resultados de ciertos acontecimientos previos (esta vez a nivel genético) se representan físicamente en la anguila, aunque tampoco esta vez sepamos cómo ni donde. Puede ser que los dos tipos de representaciones sean diferentes, pero ninguna de ellas parece más "teleológica" que la otra, puesto que ambas son físicas y deterministas. Skinner parece conceder otro tanto cuando dice en las 
dos frases siguientes: "Desde luego que debe haber algún "estado" de la anguila (¿qué otra cosa podría ser?) que la lleva a seguir las direcciones correctas. Este estado puede compararse con el que se da en un organismo que ha sido condicionado, de hecho, a realizar el mismo viaje, pero constituye el producto final de un proceso completamente diferente" (p. 72). El que sea o no un proceso diferente no nos dice nada sobre el papel del reforzamiento en la migración.

Aunque yo no he visto la migración de las anguilas, he observado a los salmones nadando contracorriente, muchas veces en cascadas, en el río Columbia, volviendo a las cabeceras poco profundas del río para desovar y morir. Este también es un viaje que se hace una sola vez en la vida, otro ejemplo de la "conducta filogenética" mencionada por Skinner (1975). No creo que esta conducta sea hedónicamente neutral, como la de "digerir la comida». El salmón hace un gran esfuerzo por remontar el río; hasta podría presionar una palanca para conseguir la oportunidad de hacerlo. En gran parte de las conductas filogenéticas parece como si hubiera mucho de refuerzo, y hipótesis era que los estímulos reforzantes surgen, en parte, de la ejecución de la propia conducta.

El carácter compulsivo afectivo y de urgencia de las conductas instintivas - para utilizar un término que se las aplicaba antes - ha sido claro para los observadores y comentadores desde hace tiempo. William James (1690), por ejemplo, decía:

"¿Por qué se acuestan los hombres, cuando pueden, en lechos blandos y no en suelos duros? ¿Por qué se sientan alrededor del hogar en los días fríos? ¿Por qué se sitúan en las habitaciones, el noventa $y$ nueve por ciento de las veces, mirando a la parte central y no a la pared? ¿Por qué prefieren el lomo de carnero y el champagne a los bizcochos y al agua de una acequia? ¿Por qué le interesa la doncella al joven hasta tal punto que todo lo que se refiere a ella le parece lo más importante y significativo del mundo? Lo más que podemos decir es que estas son formas de conducta humana y que a todas las criaturas les gustan sus propias formas de conducta, y les resulta natural seguirlas. La ciencia podrá estudiar esas conductas, tomarlas en consideración, y descubrir que la mayor parte de ellas son útiles. Pero no es por su utilidad por lo que se siguen, sino porque en el momento de efectuarlas sentimos que son lo único apropiado y natural que podemos hacer. Ni un sólo hombre entre un billón piensa en la utilidad de comer mientras come. Come porque el alimento le gusta y le hace querer comer más." (p. 386).

Si James hubiera escrito hoy hubiera dicho que a todas las criaturas «les refuerzan sus propias formas de conducta”.

Si Skinner pretende negar el valor reforzante de las conductas filogenéticas, me parece que es él el que tiene que presentar pruebas para demostrar en qué se diferencian de las conductas como comer y beber que proporcionan los reforzadores de gran parte de las investigaciones operantes. Para realizar esta tarea tendrá que enfrentarse a los descubrimientos recientes. (Cohen, 1975) que han demostrado que, por lo menos, algunas de las conductas adjuntivas de las ratas tienen un valor de reforzamiento comparable, en cuanto a sus efectos, a los reforzadores característicos de la investigación operante. Cohen demostró que la poli- 


\section{Estudios}

dipsia inducida por un programa - un ejemplo de respuesta filogenética ampliamente estudiada- obedece a la ley del emparejamiento, que es una característica muy observada de las respuestas reforzadas (de Villiers, 1977). Sería extraño que los organismos obedeciesen a la ley del efecto sólo cuando no hacen lo que resulta natural; afortunadamente, los datos no permiten sacar esta conclusión tan extraña. Debemos asegurarnos de que nuestras teorias tampoco.

Skinner encuentra defectos en varios aspectos de mi caracterización de su posicion. Es evidente que a él debemos considerarle la autoridad final sobre el tema. Sin embargo, se puede demostrar que mis caracterizaciones describían fielmente sus puntos de vista en determinados estadios de su pensamiento. Cuando alguien escribe nueve libros sobre un tema se le puede permitir que modifique un poco su posición y que no recuerde todos sus pasos. Por ejemplo, yo decía que Skinner eía pesimista eñ cuanto a lá posibilidad de unna ciencia de la conducta completamente predictiva». El lo niega. Sin embargo, esto era lo que decia sobre el tema en 1938:

"Confrontados a la gran amplitud de la topografía de la conducto, tenemos que reconocer que resulta imposible hacer una predicción general de los estímulos o respuestas que pudiera considerarse exacta. El número de elementos con el que hay que enfrentarse es muy grande y no parece probable que se reduzca $y$, hoy por hoy, no hay razones para creer que vaya a descubrirse algún día un nuevo orden para resolver la dificultad. Esta concepción puede parecerles sombría a los que creen que el estudio de la conducta se ocupa primordialmente de la predicción topográfica de los estímulos y res- puestas. Pero ésta es una caracterización errónea y fatal de su objetivo.» (p. 11).

Ahora Skinner niega haber dicho que "la única forma de atrapar el flujo de conducta (como Watson lo caracterizaba) sea botanizar los reflejos". En 1938 decía que "no hay ningún modo de lograr el objetivo establecido en esta cita (es decir, la pretensión del conductismo de Watson), tomada literalmente, que no sea botanizar» (p. 10). El problema es que el conductismo skinneriano se formuló originalmente como ciencia de laboratorio, cuyo objetivo era «la predicción de las propiedades cuantitativas de reflejos representativos» (Skinner, 1938, p. 12), y no una descripción completa de la conducta real de una criatura en particular. En cuarenta años ha pasado a la conducta real de determinadas criaturas, a saber: las personas.

En este paso hay más implicaciones de las que se dicen. El problema, en su sentido más amplio, es como pasar de las condiciones de iaboratorio completamente apropiadas en que ciertos organismos representivos manifiestan "las propiedades cuantitativas de reflejos representativos", a la conducta real en vivo. Se trata del problema familiar de la extrapolación; sería sorprendente que alguien consiguiera evitarlo en psicología. En mi artículo argumentaba que los conductistas han confundido, a veces, la relativa simplicidad de la conducta de laboratorio con la conducta que se da fuera de él, especialmente con respecto a las variables que trataba de describir.

En un período anterior de su carrera, Skinner (1938) era cauto: "dejémosles que extrapolen lo que quieran. Por ahora no podemos decidir si una extrapolación está justificada o no» (p. 442). Sus escritos posteriores no dejan lugar a dudas de que creía que estaba ampliamente justificado realizar extrapo- 
laciones a casi todos los aspectos de los problemas humanos. Creo que puede bastar con decir que yo creo que nuestras extrapolaciones a los problemas humanos serian más iluminadoras y más útiles si supiéramos más sobre los estímulos a los que responden las personas, las conductas que realizan y los reforzadores por los que se mueven, además de conocer las propiedades cuantitativas de la conducta de ciertos organismos representativos. Con ello no quiero negar ni la importancia de conocer esas propiedades cuantitativas, ni mi respeto y admiración a las profundas contribuciones que ha hecho el profesor Skinner a nuestro conocimiento de ellas.

También niega Skinner que los impulsos sean estados. En 1938 decía, refiriéndose a la respuesta de comer de las ratas: "Como las respuestas de comer no son inevitables, nos vemos obligados a proponer la hipótesis de que hay un estado interno, al que debemos asignar !a variabilidad” (p. 341). Aunque ahora desacredite esta noción, yo no puedo, honestamente, atribuirme el mérito de haber hecho la brillante clarificación del concepto de impulso que realizó Skinner en sus primeros trabajos. En los más recientes prefiere no utilizar el concepto de "impulso" ni el de "estado". Por el contrario, se limita a las operaciones que afectan a las conductas, como ocurre con la privación de comida en relación a la alimentación, y evita tratar de los estados de impulso que intervienen. La diferencia es teórica, no empr̃ica,y, por tres razones teóricas, yo prefiero el primer skinnerianismo al último en este aspecto. En primer lugar, un organismo puede estar dispuesto a realizar una determinada conducta y no tener forma de hacerlo. Por ejemplo: una rata puede tener hambre sin que haya comida. La objetividad y observabilidad de nuestros conceptos no es mayor porque digamos que la rata tiene una tendencia inexpresada a comer y no que está en un estado actual que incluye el hambre. La conducta observable no nos cuenta nunca toda la historia de la psicología de un organismo, prescindiendo de cómo llamemos a las tendencias o estados inobservados. Creo que es conveniente tener un nombre, como el de "hambre", para designar la tendencia a comer, a realizar otras actividades relacionadas con la comida y a ser reforzado por el alimento y los estímulos relacionados con él. En segundo lugar, los estados de impulso implican ciertos hechos sobre las clases de conductas y de reforzamientos, como discutía en detalle en mi artículo. Creo que si nuestra teoría tiene un nombre para ellos es menos probable que perdamos de vista lo poco que sabemos acerca de estos hechos en cualquier especie, incluyendo la nuestra. Finalmente, los impulsos forman parte de la experiencia ordinaria y han sido forjados por el conocimiento ordinario. A mi entender, es mejor dar sentido científico al conocimiento ordinario que insistir en que está en el error.

\section{Resumen}

Tal y como emplea Skinner el término «conducta filogenéticas, se refiere a un tercer tipo de conducta, además de la operante y la respondente. Aquí sugerimos que no es necesario ni deseable añadir una tercera categoría de conducta al análisis conductista. El argumento gira en torno a la conducta autorreforzante, el estatuto del impulso y en la discusión sobre si la ley del efecto se refiere al aprendizaje y la ejecución o sólo a la ejecución. 


\section{Summary}

Phylogenic behavior, as used by Skinner, refers to a third kind of behavior, in addition to operant and respondent. It is suggested here that a third category of behavior is neither a necessary nor a desirable addition to a behavioristic analysis. The argument hinges on self-reinforcing behavior, on the status of drive, and on whether the law of effect concerns both learning and performance or just performance.

\section{Resumé}

Telle qu'est employe par Skinner, l'expression econduite philogénétique» est référée à un troisième type de conduite, en plus de l'opérante et la répondante. On est suggeré ici qu'il n'est pas nécéssaire ni désirable ajouter une troisième catégorie d'ana. lyse de conduite. L'argument est axé sur la conduite autorenforçante, sur le status de la pulsion et sur la discussion de la question de savoir si la loi de l'effet concerne à l'apprentissage et à l'exécution ou seulement à l'execution.

\section{Referencias}

BROWN, R. \& HERRNSTEIN, R. J. Psychology. Boston, Little, Brown, 1975.

COHEN, I. L. The reinforcement value of schedule-induced drinking. Journal of the Experimental Analysis of Behavior, 1975, 23, 37-44.

DE VILLIERS, P. A. Choice in concurrent schedules and a quantitative formulation of the law of effect. En W. K. Honing \& J. E. R. Staddon (Eds.), Handbook of operant behavior. Englewood Cliffs, N. J. Prentice-Hall, 1977.

ESTES, W. K. New Perspectives on some old issues in association theory. En N. J. Mackintosh \& W. K. Honing (Eds.), Fundamental issues in association learning. Hallifax, Canadá, Dalhousie University Press, 1969.

HERRNSTEIN, R. J. The evolution of behaviorism. American Psychologist, 1977, 32, 593-603. JAMES, W. Principles of psychology (vol. 2). Nueva York. Holt, 1890.

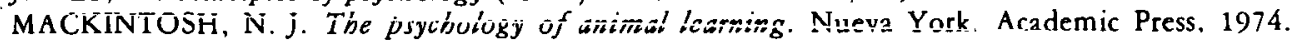
SKINNER, B. F. The bebavior of organisms. Nueva York. Appleton, 1938.

SKINNER, B. F. The shaping of phylogenic behavior. Journal of the Experimental Analysis of Behavior, 1975, 24, 117-120.

SKINNER, B. F. Herrnstein and the evolution of behaviorism. American Psychologist, 1977, 32, 1006-1012. 